\title{
AS PRÁTICAS ALIMENTARES NO PERÍODO GESTACIONAL: Uma revisão integrativa
}

\author{
Vinicius Lino de Souza Neto. Discente do Curso Bacharelado em Enfermagem da Universidade Federal de \\ Campina Grande - Campus - Cuité/Paraíba/Brasil. E-mail: vinolino@hotmail.com \\ Janaína von Söhsten Trigueiro. Enfermeira e Fonoaudióloga. Mestre em Enfermagem na Atenção à Saúde \\ pela Universidade Federal da Paraíba/UFPB/PB/BRASIL. Docente do Curso de Bacharelado em Enfermagem da \\ Universidade Federal de Campina Grande - UFCG/ Campus - Cuité. E-mail: janavs_23@hotmail.com
}

Isolda Maria Barros Torquato. Enfermeira e Fisioterapeuta. Mestre em Ciências da Nutrição pela Universidade Federal da Paraíba/UFPB/PB/BRASIL. Docente do Curso de Bacharelado em Enfermagem da Universidade Federal de Campina Grande - UFCG/ Campus - Cuité. E-mail: isoldatorquato@ig.com.br

Recebido em: 14/05/2013 - Aprovado em: 30/07/2013 - Disponibilizado em: 15/08/2013

\section{RESUMO}

Objetivo: explanar as práticas alimentares durante o período gestacional. Método: estudo exploratório e descritivo, realizado por meio de revisão integrativa da literatura, utilizando publicações científicas disponibilizadas nas bases de dados LILACS, Medline e SciELO, entre os anos de 2006 a 2012, tendo como amostra final 21 artigos. Resultados: As práticas alimentares da nutrição materna exerce grande impacto no resultado da gestação e sobre a saúde da mulher e da criança durante a lactação, onde a avaliação nutricional individualizada no início do pré-natal é importante para estabelecer as necessidades de nutrientes neste período e deve ser realizada continuamente ao longo da gravidez. Ressalta-se a importância da compreensão e valorização das questões socioculturais e econômicas que influenciam as práticas alimentares, a fim de que a orientação alimentar e nutricional, visando à alimentação saudável, possa ser negociada e ajustada às necessidades e à subjetividade das mulheres grávidas, tendo como meio articulador a educação em saúde. Conclusão: os resultados encontrados evidenciam uma pluralidade nas praticas alimentares desenvolvidas pelas gestantes, pois as mesmas são influenciadas diretamente por diversos cofatores externos, como a cultura, crença e tradições.

Descritores: Gestação. Hábitos Alimentares. Gravidez. Nutrição Materna. Educação em Saúde.

\section{ABSTRACT}

Objective: To explain the dietary practices during pregnancy. Method: exploratory and descriptive study, conducted through integrative literature, using scientific publications available in the databases LILACS, SciELO and MEDLINE between the years 2006 to 2012, with the final sample of 21 articles. Results: The dietary practice of maternal nutrition exerts great impact on the outcome of pregnancy and the health of women and children during lactation, where individualized nutritional assessment in early prenatal care is important to establish the nutrient requirements during this period and must be performed continuously throughout pregnancy. We emphasize the importance of understanding and appreciation of sociocultural and economic issues that influence eating habits, so that the food and nutrition counseling, aimed at healthy eating, can be negotiated and adjusted to the needs and subjectivity of pregnant women taking as a means articulating health education. Conclusion: The results show a plurality in dietary practices developed by pregnant women because they are directly influenced by several external cofactors, such as culture, beliefs and traditions.

Keywords: Pregnancy. Food Habits. Pregnancy. Maternal Nutrition. Education in health

\section{INTRODUÇÃO}

Compreendendo que o ciclo vital abrange o nascer, crescer, reproduzir e morrer, a mulher surge como aquela que possui a capacidade de gerar uma nova 
vida em seu ventre, ocorrendo, pela ação de diversos mecanismos intrínsecos, refletindo assim em uma série de modificações físicas, fisiológicas e psicológicas que regulam as funções orgânicas da mãe para garantir o desenvolvimento do concepto (SANTOS et al, 2011). No entanto, para que esse momento suceda normalmente, é necessário que a alimentação materna seja rica em nutrientes durante a gestação, considerando que esta será a única fonte nutricional para o feto (COTTA et al, 2009).

Assim, o estado nutricional da mulher antes e durante a gestação é um fator fortemente associado à ocorrência de complicações gestacionais como diabetes, pré-eclâmpsia, hipertensão, insuficiência cardíaca, prematuridade, retardo de crescimento uterino, defeito do tubo neural e morte neonatal (NOCHIERI, 2008). Haja vista a existência de peculiaridades no mundo feminino, sobretudo na fase da gravidez, o consumo de diferentes tipos de alimentos e os modos de se alimentar são influenciados por questões fisiológicas e emocionais, pela cultura, pela situação socioeconômica e nem sempre estão de acordo com o que afirma a ciência em relação à alimentação saudável (BAIÃO; DESLANDES, 2008).

Estudos revelam que o problema nutricional de maior prevalência no Brasil durante o período gravídico é o excesso de peso, com taxas que variam de 25 a $30 \%$. Além dos achados brasileiros, na Inglaterra e nos Estados Unidos também há proporções elevadas de mulheres em idade fértil com sobrepeso e obesidade (VITOLO; BUENO; GAMA, 2010).

Nesta perspectiva, inadequação do estado nutricional materno no período prégravídico e gravídico constitui um problema de saúde pública, pois contribui para o surgimento de intercorrências que influenciam negativamente no curso da gestação (BELARMINO et al., 2008).

Salienta-se que a avaliação nutricional de gestantes na rede básica de saúde é realizada durante as consultas de pré-natal, por enfermeiros e médicos, que seguem diretrizes recomendadas pelo Ministério da Saúde (MS) (MARTINS; BENICIO, 2011). Logo, o acompanhamento nutricional individualizado deve ser realizado por meio dessas consultas, sendo uma atividade obrigatória e, de preferência, efetivada pelo enfermeiro, o qual subsidia o trabalho do nutricionista neste âmbito (MAALTA et al, 2008).

Perante o exposto, objetivou-se analisar as produções científicas publicadas no período de 2006 a 2012, com ênfase nas práticas alimentares desenvolvidas por mulheres durante a gestação. 


\section{METODOLOGIA}

Foi realizada uma revisão integrativa da literatura, operacionalizada pelas etapas de formulação do problema, coleta de dados, avaliação dos dados, análise e interpretação dos dados coletados e apresentação dos resultados. A revisão integrativa segundo Souza, Silva e Carvalho (2010), determina o conhecimento atual sobre uma temática específica, já que é conduzida de modo a identificar, analisar e sintetizar resultados de estudos independentes sobre o mesmo assunto, contribuindo, pois,para uma possível repercussão benéfica ao tema estudado.

Ademais, possibilita a divulgação do conhecimento, pois um único estudo disponibiliza ao leitor os resultados de várias pesquisas (MENDES; SILVEIRA; GALVÃO, 2008). Convém ressaltar que o estudo teve o intuito de responder a seguinte questão norteadora: Qual a prevalência da diversificação das práticas alimentares durante a gravidez?

A identificação das publicações foi mediada, inicialmente, por meio da busca nas seguintes bases de dados indexadas na Biblioteca Virtual em Saúde (BVS): Literatura Latino-Americana e do Caribe em Ciências da Saúde (LILACS), Medical Literature Analysisand Retrieval Sistem on-line (Medline), Bancos de Dados em Enfermagem (BDENF).

No primeiro momento, utilizaramse como descritores, tanto em português como em inglês: "Gestação/Gestation”; "Gravidez/Pregnancy"; "Hábitos Alimentares/Eating Habits" "Educação em Saúde/Education in health" e "Nutrição Materna/Maternal Nutrition”. Após a seleção, os artigos foram lidos na íntegra e elencados de acordo com os seguintes critérios de inclusão: periódicos publicados em português e inglês, disponibilizados na íntegra, coerentes com a temática proposta, publicados no período entre 2006 a 2012. Durante a seleção, houve a exclusão de artigos conforme alguns critérios, respectivamente: trabalhos que abordassem outros tipos de temática, produções científicas com animais experimentais, trabalhos direcionados a crianças e àqueles que estivessem disponíveis em outro idioma.

A busca foi efetivada nos meses de janeiro á março de 2013, e as estratégias utilizadas para o levantamento dos artigos foram adaptadas para cada uma das bases de dados, de acordo com suas especificidades de acesso, tendo como eixo norteador a pergunta central e os critérios de inclusão e exclusão. A análise criteriosa das publicações possibilitou a obtenção das informações mais relevantes e, assim, 21 artigos constituíram a amostra final. 
A localização dos artigos, corpus do estudo, foi realizada, de forma independente, como estratégia de garantir a legitimidade do conteúdo de análise (POLIT; BECK; HUNGLER, 2006). A fim de analisar os dados utilizou-se a análise textual, a qual possibilita o aprofundamento nos processos discursivos, visando alcançar saberes sob a forma de compreensões reconstruídas dos discursos. Esse método de análise permite identificar e isolar enunciados dos conteúdos a ele submetidos, categorizar tais enunciados e produzir textos, de maneira a integrar descrição e interpretação. A análise textual utiliza como fundamento de sua construção o sistema de categorias, o corpus conjunto de textos submetidos à apreciação, que representa a multiplicidade de visões de mundo dos sujeitos acerca do fenômeno investigado (MORAES, 2005).

Convém ressaltar que foram consideradas as diretrizes éticas contidas na resolução 311/2007 do Conselho Federal de Enfermagem na qual o CEPE é regulamentado em especial ao que concerne aos capítulos III - Do ensino da pesquisa e da produção técnico cientifica; e IV - Da publicidade.

\section{RESULTADOS}

Os dados a seguir são resultantes do levantamento realizado na literatura, de acordo com os critérios de seleção elaborados pelos pesquisadores para a realização da análise do material. No processo de operacionalização, foram elencadas as variáveis como ano e idioma, conforme explana a Tabela 1.

Tabela 1 - Distribuição das publicações da área da saúde com enfase das práticas alimentares no período gestacional entre o ano de 2006 a 2012, quanto ao ano e idioma.

Variáveis

\begin{tabular}{l|l|l}
\hline Ano de publicação & N & $\%$ \\
\hline 2006 & 03 & 14,28 \\
2007 & 01 & 4,76 \\
2008 & 05 & 23,80 \\
2009 & 04 & 19,04 \\
2010 & 02 & 9,56 \\
2011 & 05 & 23,80 \\
2012 & 01 & 4,76 \\
Total: & 21 & 100 \\
Idioma & & \\
Português & 17 & 80,95 \\
Inglês & 04 & 19,05 \\
Total: & 21 & 100 \\
\hline
\end{tabular}

Fonte: Dados da Pesquisa, 2013.

Ao analisar a Tabela 1, observa-se uma distribuição heterogênea de publicação conforme o ano, uma vez que o ano de 2006 possuiu 14,28\% (3) publicações na área investigada, em 2007 houve somente uma publicação $(4,76 \%)$, o ano de 2008 obteve 23,80\% (5), 19,04\% (4) das publicações encontrava-se em 2009, 9,56\% (2) em 2010, 23,80\% (5) em 2011 e 4,76\% (1) no ano de 2012. Quanto ao idioma, verificou-se que $80,95 \%$ (17) 
estavam disponíveis em português e $19,05 \%$ (4) em inglês.

Tabela 2 - Distribuição das publicações da área da saúde com enfase das práticas alimentares no período gestacional entre o ano de 2006 a 2012, quanto ao tipo de metodologia utilizada. Variável

\begin{tabular}{l|l|l}
\hline Tipo de Metodologia & $\mathrm{N}$ & $\%$ \\
\hline Revisão da Literatura & 04 & 19,04 \\
Transversal exploratório e & 05 & 23,82 \\
descritivo & & \\
Coorte & 03 & 14,29 \\
Retrospectivo descritivo & 03 & 14,29 \\
Caso controle & 02 & 9,52 \\
Estudo de Campo & 02 & 9,52 \\
Randomizado & 02 & 9,52 \\
Total: & 21 & 100 \\
\hline
\end{tabular}

Fonte: Dados da Pesquisa, 2013.

A Tabela 2 demonstra o caminho metodológico utilizado nas publicações, onde o estudo transversal exploratório e descritivo obteve maior percentual 23,82\%(05), seguido de 19,04\% (04) pela revisão da literatura, depois coorte e retrospectivo descritivo 14,29\% (03), e, por fim, caso controle, estudo de campo e randomizado com 9,52\% (02) totalizando o quantitativo da amostra.

Tabela 3 - Distribuição das publicações da área da saúde com enfase das práticas alimentares no período gestacional entre o ano de 2006 a 2012, quanto aos autores.

Variável

\begin{tabular}{l|l|l}
\hline Autores da Publicação & $\mathrm{N}$ & $\%$ \\
\hline Enfermeiros & 06 & 28,57 \\
Educadores físicos & 02 & 9,53 \\
Nutricionista & 09 & 42,86
\end{tabular}

\begin{tabular}{l|l|l}
$\begin{array}{l}\text { Médico (a) } \\
\text { Total: }\end{array}$ & 04 & 19,04 \\
& 21 & 100 \\
\hline
\end{tabular}

Fonte: Dados da Pesquisa, 2013.

Observando a Tabela 3 é visto que os pesquisadores que investigaram sobre a importância da diversificação alimentar no período gestacional possuem as seguintes profissões: nutricionista 42,86 (09), enfermeiro (a) 28,57 (06), médico (a) 19,04\% (04) e educadores físicos 9,53\% (02). Percebe-se que a área é mais explorada pelos nutricionistas, o que já era esperado.

\section{DISCUSSÃO}

No Brasil, a articulação das políticas publica desenvolvida pelo Ministério da Saúde (MS) preconiza a compreensão dos múltiplos significados da gestação para a gestante, assim como do contexto em que a mesma está inserida, onde o prénatal torna-se um momento privilegiado para discutir e esclarecer questões que são únicas para cada mulher (FAZIO et al, 2011). Neste sentido, Barros e Victoria (2008) apontam que assistência ao pré-natal inclui não só o acompanhamento e monitoramento de peso gestacional e sim orientações nutricionais no período que compreende da gravidez à amamentação de forma enfatica, pois conforme os princípios gerais e de condições para o acompanhamento prénatal estabelecidos na Portaria GM/MS n. 
569, a primeira consulta de pré-natal deve acontecer até $\mathrm{o} 4^{\circ}$ mês de gestação, devendo ser, no mínimo 6 (seis) consultas de acompanhamento; preferencialmente, uma no primeiro, duas no segundo e três no terceiro trimestre da gestação, estas decorrente da necessidade fisiológica da gestante.

$\begin{array}{lccc}\text { Para } & \text { Santos et al (2011) a } \\ \text { prevalência } & \text { de uma alimentação } \\ \text { diversificada } & \text { durante o processo } \\ \text { gestacional é substancial para o }\end{array}$
desenvolvimento das funções fisiológicas do binômio mãe e filho,o período gestacional possui uma conformação heterogênea em se tratando dos seus aspectos fisiológicos, metabólicos e nutricionais e com isso a gestante tende a sofrer mudanças alimentares decorrentes das mesmas (NOCHIERI et al, 2008).

Dentro deste contexto, a cultura alimentar ao qual se faz presente no cotidiano do individuo, interferem diretamente na gravidez, estas influências culturais duplicam conforme as crenças e prescrições da localidade, como por exemplo, muitas pessoas em diferentes sociedades ainda crêem que os desejos da gestante precisam ser atendidos, caso contrário a criança nascerá com alguma marca; que a coloração de alguns alimentos pode manchar a pele do bebê e que alimentos "quentes" podem provocar aborto. (BAIÃO; DESLANDES, 2006).
De acordo com Baião e Deslandes (2010), durante a gestação é indispensável o cuidado nutricional, independente dos fatores culturais,se detendo a uma atenção com as variáveis orgânicas, de modo que seja possível a identificação de gestações potencialmente de risco, no entanto todo o trabalho de educação nutricional torna-se mais lento se iniciado somente no período de gestação. Assim, é fundamental de que certos cuidados sejam iniciados ainda no período pré-concepção, dentre os quais se destaca o acompanhamento nutricional das mulheres que desejam engravidar ou ainda no primeiro contato com a gestante, se estendendo ao longo das consultas pré natais, (SILVA et al,2007).

As orientações nutricionais devem ser oferecidas de acordo com as possibilidades econômicas, sociais e culturais de cada paciente, o que implica na necessidade de adequado preparo dos profissionais da área da saúde em relação ao assunto, conforme retrata Giacomello et al, (2008). Neste mesmo pensamento lógico, os profissionais de saúde, ao qual trabalham no contexto de assistência, podem assumir importante papel na educação em saúde, no incentivo às gestantes quanto aos aspectos de hábitos saudáveis de vida e nutricionais, na identificação de gestantes em risco nutricional, através da avaliação do estado 
nutricional, assim como encaminhar as gestantes para programas de assistência social quando diagnosticadas situações de necessidade (LEANDRO et al ,2009).

Baião e Deslandes (2008) discute que o ser humano, além de escolher aquilo que come, considera outros aspectos inerentes ao ato alimentar, como as distintas formas de preparo e preservação de alimentos, as quantidades, os horários, os momentos especiais, os locais e a comensalidade. Esse conjunto de itens compõe diferentes práticas alimentares que se relacionam às experiências socioculturais e às representações coletivas de forma introspectiva (CASTRO; KAC; SICHIERI, 2009).

A adequação da assistência prénatal do ponto de vista qualitativo pressupõe a atuação de profissionais de saúde preparados para identificar gestantes em risco nutricional, através da avaliação do estado nutricional precoce, assim como realizar orientação nutricional individualizada visando à otimização do estado nutricional materno, a melhoria das condições maternas para o parto e a adequação do peso do recém-nascido (COTTA et al,2009; SANTOS; MAMEDE; BERNARDI, 2006).

Dessa forma, uma orientação contribui para a formação de gestantes conscientes e preparadas para lidar com os fatores vulneráveis, contudo torna-se necessário à implementação de estratégias de atenção à saúde direcionadas a esse grupo populacional referente ao cuidado nutricional, levando em consideração os costumes e a cultura alimentar local, visando a contribuir para a melhoria da qualidade de vida e promoção da saúde.

\section{CONSIDERAÇÕES FINAIS}

Após os artigos revisados, entendemos que os resultados encontrados evidenciam uma pluralidade nas praticas alimentares desenvolvidas pelas gestantes, pois as mesmas são influenciadas diretamente por diversos cofatores externos, como a cultura, crença e tradições, mas hoje conforme o conteúdo cientifico da pesquisa a preocupação está tangenciada mais ao sobrepeso da grávida, esquecendo um pouco os casos de baixo peso, por isso, que ainda, a necessidade de discussão e reivindicação por políticas de saúde favoráveis à distribuição de alimentos e suplementos às gestantes necessitadas.

Ressalta-se ainda, com está revisão literária, a importância de teor substancial que é a pratica da educação em saúde desenvolvido por profissionais qualificados voltados para a diversificação alimentar durante a gestação e como são deficientes as políticas assistências a gestantes. Com isso, acreditamos que as gestantes estão a 
merecer o apoio no que se refere à adequação nutricional, tanto dos profissionais de saúde como dos detentores do poder.

Faz-se necessária a adequação da assistência nutricional para que assim venha a transformar o discurso em prática real. Desta forma, o profissional de saúde deve agir na minimização de fatores que acarretem hábitos maléficos, compreendendo o aspecto sociocultural presente na prática alimentar, e considerando-o no processo de modificação continuada e transformadora do hábito alimentar do indivíduo. Devem ser incentivadas ações de cuidados especiais voltados para as gestantes, ou seja, campanhas informativas que levem a

\section{REFERÊNCIAS}

BAIÃO, M.R.; DESLANDES, S.F. Gravidez e comportamento alimentar em gestantes de uma comunidade urbana de baixa renda no Município do Rio de Janeiro, Brasil. Cad. Saúde Pública. 2008. 24(11): 2633-2642. Disponível em:

http://www.scielo.br/scielo.php?pid=S0102-

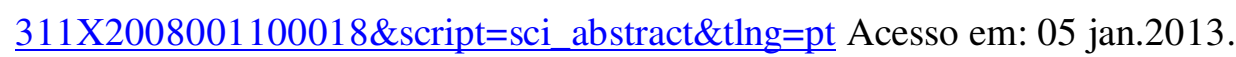

BAIÃO, M.R; DESLANDES, S.F. Alimentação na gestação e puerpério. Revista de Nutrição. 2006. 19(2): 245-253. Disponível em:

http://www.scielo.br/scielo.php?script=sci_arttext\&pid=S1415-52732006000200011 Acesso em: 05 jan. 2013.

BAIÃO, M.R; DESLANDES, S.F. Práticas alimentares na gravidez: um estudo com gestantese puérperas de um complexo de favelas do Rio de Janeiro (RJ, Brasil). Ciência \& Saúde Coletiva. 2010.15(Supl. 2): 3199-3206. Disponível em: 
http://www.scielo.br/scielo.php?script=sci_arttext\&pid=S1413-81232010000800025 Acesso em: 05 jan.2013.

BARROS, F.C; VICTORIA, C.G. Saúde materno-infantil em Pelotas, Rio Grande do Sul, Brasil: principais conclusões das comparações de coortes de nascimento de 1982, 1993 e 2004. Cad. Saúde Pública. 2008. 24 Sup 3:S461-S467. Disponível em:

http://www.scielo.br/scielo.php?pid=S0102-311X2008001500012\&script=sci_arttext Acesso em: 05jan.2013.

BELARMINO, G.O et al. Risco nutricional entre gestantes adolescentes. Acta Paul Enferm. 2009. 22(2): 169-75. Disponível em: http://www.scielo.br/pdf/ape/v22n2/a09v22n2.pdf Acesso em: 05 jan.2013.

CASTRO, M.B.T; KAC, G; SICHIERI, R. Determinantes Nutricionais e sócio-demográficos da variação de peso no pós- parto: uma revisão da literatura. Rev Bras. Saud. Materna Infantil. 2009. 9(2): 125-137. Disponível em:

http://www.scielosp.org/scieloOrg/php/related.php?pid=S0102-

$\underline{311 X 2001000300002 \& l a n g=e n ~ A c e s s o ~ e m: ~ 05 j a n .2013 . ~}$

COTTA, R.M.M et al. Aspectos relacionados aos hábitos e práticas alimentares de gestantes e mães de crianças menores de dois anos de idade: o programa saúde da família em pauta. $\mathrm{O}$ Mundo da Saúde. 2009. 33(3): 294-302. Disponível em: http://www.saocamilosp.br/pdf/mundo_saude/69/294a302.pdf Acesso em: 05 jan.2013.

FAZIO, E.S et al. Consumo dietético de gestantes e ganho ponderal materno após aconselhamento nutricional. Rev Bras Ginecol Obstet. 2011.33(2): 87-92: Disponível em: http://www.scielo.br/pdf/rbgo/v33n2/v33n2a06.pdf Acesso em: 05 jan.2013.

GIACOMELLO, A et al. Validação relativa de questionário de frequência alimentar em gestantes usuárias de serviços do Sistema único de Saúde em dois municípios do Rio Grande do Sul, Brasil. Rev Bra. Saúde Mater. Infant. 2008. 8(4): 445-454. Disponível em: http://www.scielo.br/scielo.php?pid=S1519-38292008000400010\&script=sci_arttext Acesso em: 05 jan.2013. 
LEANDRO, C.G et al. Nutrição, programação fetal e atividade física. Revista de Nutrição. 2009. 22(4): 559-569. Disponível em: Acesso em:

MAALTA, M.B et al. Utilização das recomendações de nutrientes para estimar prevalência de consumo insuficiente das vitaminas C e E. Rev Bras Epidemiol. 2008.11(4): 573-83.

Disponível em: http://www.scielo.br/scielo.php?pid=S1415-

790X2008000400006\&script=sci_abstract\&tlng=pt Acesso em: 08 jan.2013.

MARTINS, A.P.B; BENICIO, M.H.D.A. Influência do consumo alimentar na gestação sobre a retenção de peso pós-parto. Rev Saúde Pública.2011. 45(5): 870-77. Disponível em: http://www.scielo.br/scielo.php?pid=S0034-89102011000500008\&script=sci_arttext Acesso em: 08 jan.2013.

MORAES, R. Mergulhos discursivos: análise textual qualitativa entendida como processo integrado de aprender, comunicar e interferir em discursos. In: Gagliase MC, Freitas JV, organizadores. Metodologias emergentes de pesquisa em educação ambiental. Ijuí: Ed Unijuí; 2005. p. 85-114.

MENDES,K.D.S; SILVEIRA, R.C.C.P; GALVÃO, C.M. Revisão integrativa: métodode pesquisa para a incorporação de evidências na saúde e na enfermagem. Texto Contexto Enferm. 2008.17(4): 758-64.Disponível em: . http://200.144.190.38/bitstream/handle/2012.1/3509/art_MENDES_Revisao_integrativa_met odo_de_pesquisa_para_a_2008.pdf?sequence=1. Acesso em: 05 jan.2013.

NOCHIERI, A.C.M et al. Perfil Nutricional de gestantes atendidas em primeira consulta de Nutrição no Pré-Natal de uma instituição filantrópica de São Paulo. O Mundo da Saúde. 2008. 32(4): 443-451. Disponível em: http://www.saocamilosp.br/pdf/mundo saude/65/05 Perfil baixa.pdf Acesso em: 08 jan.2013.

POLIT, D.F; BECK, C.T; HUNGLER, B.P. Essentials of nursing research: methods, appraisal and utilization. Philadelphia: Lippincott Williams \& Wilkins; 2006. Using research in evidence-based nursing practice; p. 457-94. 
SANTOS, E.V.O et al. Estado Nutricional Pré-Gestacional e Gestacional: uma Análise de Gestantes Internas em um Hospital Público. R bras ci Saúde. 2011. 15(4): 439-446.

Disponível em: http://periodicos.ufpb.br/ojs/index.php/rbcs/article/view/10028 Acesso em: 08 jan.2013.

SANTOS, L.A; MAMEDE, F.V; BERNARDI, J.V.B. Orientação nutricional no pré-natal em serviços públicos de saúde nomunicípio de ribeirão preto: o discurso e a prática assistencial. Rev Latino-am Enfermagem. 2006. 14(5). Disponível em:

http://www.scielo.br/scielo.php?pid=S0104-11692006000500008\&script=sci_arttext\&tlng=pt Acesso em: 08 jan.2013.

SOUZA, M.T; SILVA, M.D; CARVALHO, R. Revisão integrativa: o que é e como fazer. Einstein. 2010; 8(1 Pt 1):102- 6. Disponível em:

http://apps.einstein.br/revista/arquivos/PDF/1134-Einsteinv8n1_p102-106_port.pdf Acesso em: 08 jan.2013.

SILVA, L.S.V et al. Micronutrientes na gestação e lactação. Rev. Bras. Saúde Matern. Infant. 2007. 7 (3): 237-244.Disponível em:

http://www.nescon.medicina.ufmg.br/biblioteca/imagem/0043.pdf Acesso em: 08 jan.2013.

VITOLO, M.R; BUENO, M.S.F; GAMA, C.M. Impacto de um programa de orientação dietética sobre a velocidade de ganho de peso de gestantes atendidas em unidades de saúde. Rev Bras Ginecol Obstet.2011.33(1): 13-9. Disponível em: http://www.scielo.br/pdf/rbgo/v33n1/a02v33n1.pdf Acesso em: 10 jan.2013. 